Mens

revue d'histoire intellectuelle de l'Amérique française

MENS

\title{
Gilles Leclerc, un inquisiteur oublié
}

\section{Gilles Labelle}

Volume 3, numéro 2, printemps 2003

Regards sur la Révolution tranquille

URI : https://id.erudit.org/iderudit/1024643ar

DOI : https://doi.org/10.7202/1024643ar

Aller au sommaire du numéro

Éditeur(s)

Centre de recherche en civilisation canadienne-française

ISSN

1492-8647 (imprimé)

1927-9299 (numérique)

Découvrir la revue

Citer cet article

Labelle, G. (2003). Gilles Leclerc, un inquisiteur oublié. Mens, 3(2), 193-231. https://doi.org/10.7202/1024643ar

\section{Résumé de l'article}

La pensée de Gilles Leclerc (1928-1999) n’a jamais été véritablement reçue dans les milieux intellectuels et littéraires québécois. Pourtant, le Journal d'un inquisiteur, publié en 1960 (et réédité en 1974 et 2003), constitue un ouvrage qui a certainement sa place dans l'histoire des idées au Québec. Gilles Leclerc y expose une conception du passage de la société québécoise à une forme de modernité radicale qui accorde une grande place aux effets imprévus engendrés par ce qu'il nomme le "système ethno-théologico-politique ". Pour lui, la liberté nouvelle qu'il sent poindre à l'aube de la Révolution tranquille se confond avec la « licence » et ne pourra ainsi conduire qu’à une société fondée sur l'utilitarisme et l'hédonisme. Au bout du « système ethno-théologico-politique », en somme, se profile l'« intégration pan-américaine ", assortie d'une tentation " nihiliste " (première partie). Cette conception s'appuie sur une anthropologie et une philosophie de l'histoire cohérentes et très élaborées, qui reposent sur une analyse des rapports qui doivent s'établir entre l'« Esprit » et l'« Histoire » (deuxième partie).
Ce document est protégé par la loi sur le droit d'auteur. L'utilisation des services d’Érudit (y compris la reproduction) est assujettie à sa politique d'utilisation que vous pouvez consulter en ligne.

https://apropos.erudit.org/fr/usagers/politique-dutilisation/ 


\title{
GILLES LECLERC, UN INQUISITEUR OUBLIÉ ${ }^{1}$
}

\author{
Gilles Labelle \\ Département de science politique \\ Université d'Ottawa
}

\section{RÉSUMÉ}

La pensée de Gilles Leclerc (1928-1999) n’a jamais été véritablement reçue dans les milieux intellectuels et littéraires québécois. Pourtant, le Journal d'un inquisiteur, publié en 1960 (et réédité en 1974 et 2003), constitue un ouvrage qui a certainement sa place dans l'histoire des idées au Québec. Gilles Leclerc y expose une conception du passage de la société québécoise à une forme de modernité radicale qui accorde une grande place aux effets imprévus engendrés par ce qu'il nomme le «système ethno-théologico-politique ». Pour lui, la liberté nouvelle qu'il sent poindre à l'aube de la Révolution tranquille se confond avec la « licence » et ne pourra ainsi conduire qu'à une société fondée sur l'utilitarisme et l'hédonisme. Au bout du "système ethno-théologico-politique ", en somme, se profile l'" intégration pan-américaine », assortie d'une tentation " nihiliste » (première partie). Cette conception s'appuie sur une anthropologie et une philosophie de l'histoire cohérentes et très élaborées, qui reposent sur une analyse des rapports qui doivent s'établir entre l'« Esprit » et l'« Histoire » (deuxième partie).

\section{ABSTRACT}

Gilles Leclerc's (1928-1999) thought was never truly accepted in Quebec's intellectual and literary circles. However, bis Journal d'un inquisiteur, which was first published in 1960 and was subsequently re-edited in 1974 and 2003, deserves a place in the history of French Canadian ideas. In this work, Leclerc offers a radical interpretation of Quebec's modernisation that confers an impor- 
tant place to the unpredictable effects of what he refers to as the "systeme ethnothéologico-politique." In his mind, the new freedom granted by Quebec's nascent Quiet Revolution was notbing more than licentiousness and would inevitably lead to a society based on hedonism and utilitarianism. The ultimate conclusion of the "système ethno-théologico-politique" was pan-American integration and nibilistic temptation. Overall, Leclerc's reading of the evolution of Quebec's society was based on a coherent anthropological and historical philosophy that rested on an analysis of the relationship that should exist between "I'Esprit" and "Histoire."

Gilles Leclerc fait partie de ces penseurs dont la mémoire québécoise n'a pour ainsi dire pas conservé la trace ${ }^{2}$. Plus encore, sa pensée n'a jamais fait l'objet d'une réception à proprement parler. L'auteur a lui-même relaté ses difficultés à être publié. Le Journal d'un Inquisiteur, son ouvrage le plus important et le plus connu, est paru en 1960 aux Éditions de l'Aube, mais aux frais de l'auteur ${ }^{3}$. Au moins deux autres ouvrages de Leclerc ont été refusés par des éditeurs : L'étreinte du néant, roman " écrit dans la veine célinesque », en 1951, et Les grands cocus ("Canada Forever... Less »), en 19604. Après leur publication, les écrits de Leclerc ont été très peu discutés. Si André Major de Parti pris en a parlé favorablement ${ }^{5}$, la pensée de Leclerc était beaucoup trop éloignée de la pensée de la décolonisation pour que celle-ci se l'approprie sans malentendu. Quand le Journal d'un inquisiteur sera cité plus tard dans des ouvrages traitant de l'essai québécois, son ton polémique lui vaudra d'être considéré comme une curiosité datée, propre au Québec duplessiste ${ }^{6}$ Quelques commentateurs y verront, dans le même sens, un livre annonciateur de la Révolution tranquille ou du mouvement souverainiste ${ }^{7}$. Seul Jean Marcel, qui y percevra le «non le plus global qui ait jamais été proféré à la face non seulement du Québec comme pays, mais de ses assises historiques et spirituelles ", relèvera, sans y insister cependant, 
le fait que l'ouvrage, bien qu'il appartienne au genre de l'essai, repose sur une anthropologie cohérente et très élaborée $e^{8}$.

La teneur de cette anthropologie et de la philosophie de l'histoire dont, soutiendrai-je, elle est indissociable, est propre à faire comprendre au moins en partie l'absence de réception de l'œuvre de Gilles Leclerc. Le Journal d'un inquisiteur, en particulier, défend en 1960 des thèses d'un pessimisme radical non seulement quant à l'avenir du Québec mais également quant à celui du monde moderne tout entier (sans jamais, par ailleurs, embrasser une forme quelconque de conservatisme ou d'antimodernisme réactionnaire) ${ }^{9}$. Située par là en marge des pensées ou philosophies plus ou moins émancipatrices et optimistes en vogue au Québec dans les années 1960-70, l'œuvre de Gilles Leclerc appelle à être relue afin d'être inscrite dans la trame de la pensée québécoise tout en lui conservant sa spécificité $^{10}$. À l'heure où notre horizon culturel ou spirituel parait défini en grande partie par la déliquescence des finalités ou $l^{1}$ «anti-normativité ${ }^{11}$ » inhérente à un monde fondé sur le "prométhéisme ${ }^{12}$ » et l'« intégration pan-américaine ${ }^{13}$ ", dont Leclerc avait, l'un des premiers, averti de la menace qu'ils représentaient pour la société québécoise, il se pourrait même que cette pensée soit, paradoxalement, bien plus actuelle maintenant qu'en 1960 (ou 1974, date de la réédition du Journal).

Je procéderai en deux temps. D'abord, je chercherai à comprendre la critique de la société québécoise que propose Gilles Leclerc. Au moment de la publication du Journal d'un inquisiteur cette société est encore tout entière sous la coupe de ce que son auteur appelle un régime ou un "système ethnothéologico-politique ${ }^{14} »$. Ensuite, je chercherai à tracer les contours de l'anthropologie et de la philosophie de l'histoire, où se repère un fil conducteur que l'on pourrait qualifier globalement de "personnaliste », à laquelle cette critique renvoie. 


\section{Du « régime ethno-théologico-politique » au nihilisme}

\section{Le "régime ethno-théologico-politique »}

De prime abord, il peut sembler que ce qu'écrit Gilles Leclerc du Québec de la fin des années 1950 est peu original en ce qu'il participe de la doxa partagée par la plupart des opposants au duplessisme. Celui-ci, selon Leclerc, doit être considéré comme un épisode particulier d'un régime dont les origines remontent à la Conquête et à ses suites et dont on a déjà dit qu'il était qualifié d'« ethno-théologico-politique ». Ce qui est davantage original chez Leclerc et qui le sépare des autres opposants au duplessisme apparait quand est pris en compte le rapport qu'il établit entre ce régime et ce qu'on pourrait appeler la modernité québécoise (même si lui-même n'utilise pas ce terme). Loin d'avoir empêché l'avènement de la modernité au Québec, le régime ethno-théologico-politique est précisément ce qui l'a fait advenir. En ce sens, les thèses de Leclerc se situent à l'écart tant de la thèse classique voulant que le duplessisme ait représenté une forme de conservatisme ayant retardé la «modernisation » du Québec que de la thèse inspirée du « révisionnisme historique » voulant qu'il ait constitué un régime au fond à peu près aussi « libéral » que les régimes que l'on pouvait trouver dans des pays comparables au même moment. Pour Leclerc, comme on le verra, ce sont les aspects les plus archaïques du régime qui s'est élaboré après la Conquête et l'échec de 1837-1838 qui ont contribué, par leur logique même, à l'avènement d'une modernité radicale. C'est ce qui, selon lui, empêche de considérer dans l'abstrait la liberté qui émerge à la fin du duplessisme. Les manifestations de cette liberté nouvelle tirent leurs origines de l'ère de corruption généralisée qu'a induite le régime ethno-théologico-politique et 
qui, en définitive, a précipité sa chute. Cela interdit tout optimisme quand on envisage l'avenir de la société québécoise.

Comment définir la condition des Canadiens français à la fin des années $1950^{15}$ ? Si certains croient que cette condition revêt les apparences d'une " tragédie », en réalité il faudrait dire qu'elle relève plutôt du " pur burlesque » voire du "grotes$q^{16} e^{16} »$. D'abord, les Canadiens français sont des vaincus, des conquis. Tout, chez eux, est conditionné par « la blessure de $1759^{17}$ ». Leurs historiens leur ont raconté que tout le temps passé depuis cette date tient à une sorte de «miracle de la survivance ${ }^{18} »$. Cependant, Leclerc soutient que ce soi-disant « miracle » ne recèle aucun mystère : "c'est leur pieuse servilité qui les a en grande partie sauvés de l'extermination ${ }^{19} »$. L'Église catholique, et là réside le «burlesque » ou le " grotesque » de la chose, n'a eu de cesse qu'elle n'ait transformé dans son discours les vaincus en vainqueurs, de nourrir un absurde « complexe de supériorité » des Canadiens français dans l'univers de l'anglais, du protestantisme et du matérialisme ${ }^{20}$. Le Canada français n'est en ce sens rien d'autre qu'une scène où il s'exhibe à lui-même et admire le "chef-d'œuvre » qu'il croit être : "il se rend un culte éleusien, il devient son propre mythe $»^{21}$. Les « comédiens » sont "stupides » et les « spectateurs », qui sont les mêmes, sont "stupéfaits » : le peuple canadien-français nait du partage d'un récit fondateur constamment répété suivant lequel il rachète son expulsion de l'histoire, son expulsion de tout commencement digne, en édifiant et en se logeant dans un «ailleurs » imprenable, rien de moins qu'une Cité du Ciel où Dieu, qui n'a pas voulu la Conquête pour rien, l'a par sa Grâce installé. Ce peuple de vaincus est ainsi comiquement convaincu « de son origine céleste », il partage l'« illusion juive du peuple élu ${ }^{22} »$ - tout en étant, au contraire des Israélites, totalement dénué de l'humour qui lui permettrait de prendre une distance à l'égard de lui-même et de son " élection ${ }^{23}$ ». 
Cette manière d'habiter le monde se traduit en définitive dans l'établissement d'un régime ethno-théologico-politique, c'està-dire d'un vivre-ensemble qui s'ancre dans des références constamment rappelées à la "race française » et à l'« élection divine ». "Tout le problème des Canadiens français est $\operatorname{là}^{24}$ ", affirme Leclerc.

Redisons-le, cette manière de concevoir le Canada français n'a pas le privilège de l'originalité. Cette idée que l'histoire du Canada français a été déterminée par la Conquête, par la domination cléricale et par la "survivance ", fut une sorte de lieu commun d'une certaine pensée historique au Québec jusqu'à récemment. Mais Leclerc se démarque radicalement de ceux avec qui il partage cette vision de l'histoire du Canada français quand il traite des suites induites par le régime ethnothéologico-politique. Sa thèse est, somme toute, fort simple : ce régime a par sa logique même engendré l'inverse de ce qu'il visait. Bien loin d'avoir empêché le Québec d'accéder à la modernité, il l'y a ni plus ni moins propulsé. Bref, la modernité québécoise, plutôt que de naître d'une rupture avec l'Ancien Régime, en serait le produit achevé. Le régime ethnothéologico-politique, loin de "christianiser " ou de «catholiciser » la société québécoise - celle-ci n'aurait jamais été une société chrétienne ou catholique, - a engendré à son terme une forme radicale d'athéisme. Par là, Leclerc veut d'abord faire entendre qu'ayant provoqué une véritable « usure spirituelle ${ }^{25}$ ", le régime ethno-théologico-politique a suscité un épuisement, qui n'en laisse plus rien subsister, des valeurs françaises et catholiques sur lesquelles il prétendait s'appuyer. Ce régime a finalement engendré un climat de scepticisme envers quelque valeur que ce soit et a conduit à une forme radicale de matérialisme et d'utilitarisme. Dès lors, le régime ethnothéologico-politique a puissamment contribué, même s'il vi- 
sait le contraire, à favoriser l'intégration sans accroc de la société québécoise à l'ensemble capitaliste nord-américain.

Examinons d'abord la référence à la « race française » et, plus globalement, à la France. Certes, précise Leclerc, il n'est question ici que d'une « certaine image de la France pour consommation paroissiale ", de la France d'Ancien Régime, celle des Louis Veuillot ou Charles Maurras, servant avant tout d'alibi à la « médiocrité » et à une « incurable stérilité intellectuelle ${ }^{26}$ ». Cependant, cette référence constamment répétée à la France suscite un effet inattendu : elle pousse les opposants au régime ethno-théologico-politique à affirmer, confrontés à cette francophilie sélective, les droits de la culture canadienne-française contre la culture française. Le poids de la France est en effet tel qu'il est considéré comme une menace pour « la fête de la culture au Canada français ${ }^{27} \gg$. Les opposants - y compris Leclerc lui-même - sont ainsi amenés à clamer haut et fort que " la culture française n'est et ne sera jamais celle du Québec », que la « littérature française est une littérature étrangère pour le Québec français ${ }^{28} »$. En somme, le régime ethno-théologicopolitique conduit inéluctablement à des manifestations de francophobie $^{29}$. Ce faisant, il devient sinon impossible, du moins extrêmement difficile de tenter de s'approprier ce qui est vrai dans l'« esprit français » que Leclerc associe à l'universel afin de l'incarner en ce lieu spécifique qu'est le Québec et de le doter "d'une nouvelle qualité, d'une nouvelle dimension, la dimension américaine ${ }^{30} »$. En somme, la référence constante du régime ethno-théologico-politique à la France, " exécrable à la $\mathrm{fin}^{31}$ ", a une double conséquence : d'abord, elle retire, du fait de son invocation continue, toute valeur à la référence ellemême ; ensuite, et par le fait même, elle facilite l'orientation du Québec vers la nord-américanité. Le régime ethno-théologicopolitique contribuerait ainsi à l'engendrement d'une sorte de monstre : une société française qui, alors même qu'elle est iso- 
lée dans l'espace anglophone nord-américain vers lequel elle s'oriente de plus en plus, haïrait la France et la culture française plus que nulle part ailleurs sur le continent.

Si la référence à la France et à la « race française » a pour effet de miner l'influence de l'esprit français, l'ancrage constamment réitéré du régime dans le catholicisme a des conséquences semblables selon Leclerc. Le processus dont il est ici question est au point de départ du même ordre que le précédent : à force d'invoquer la référence catholique, on finit par l'épuiser complètement, par la vider de tout son sens, comme si « en divinisant les structures » on en venait inéluctablement à " athéise [r] l'homme ${ }^{32}$ ». En effet, en invoquant les "valeurs absolues $^{33}$ » à propos de tout et de rien, la « théocratie ${ }^{34}$ » régnante associe et identifie ces valeurs à des politiques et à des institutions qui ne sont pourtant qu'« humaines, trop humaines » (pour parler comme Nietzsche) : "les transcendances morales et spirituelles deviennent [alors] de simples instruments politiques entre les mains de l'ambition temporelle ${ }^{35} »$. Dès lors, la subordination des valeurs catholiques à des principes qui leur sont inférieurs non seulement démontre qu'elles ne sont en rien « absolues » mais conduit aussi à en faire des valeurs au sens platement utilitaires du terme, c'est-à-dire des références dont on peut disposer à sa guise, au moment jugé opportun, ce qui « équivaut à [les] détruire ${ }^{36}$ ».

Le type de personnalité ou d'être que produit à terme le régime théologico-politique (type qui, selon Leclerc, se trouvera assurément disposé au cœur de la société québécoise de l'après-catholicisme) doit être compris à partir de cette logi$q^{2} e^{37}$. Cet être sera, grosso modo, édifié sur deux socles. Le premier est l'anticatholicisme militant : loin d'avoir engendré un porteur de vertus chrétiennes, craignant le péché, cherchant à plaire à Dieu, l'Église catholique, qui prépare ainsi inlassablement "sa propre destruction ${ }^{38}$ ", a contribué à la naissance 
d'un être radicalement "désacralisé » qui n’a de cesse de dénoncer l'hypocrisie des ecclésiastiques et de proclamer la haine qu'il ressent envers eux ${ }^{39}$. Comme dans le cas de la France, que le Canada français a été poussé à rejeter alors qu'elle faisait partie de son héritage, il ne restera bientôt plus rien dans la société québécoise que l'on pourra espérer conserver du catholicisme : cette part du Canada français lui devient de plus en plus étrangère et il ne peut plus la concevoir autrement que comme une oppression qu'on lui a imposée de l'extérieur, qu'il faut effacer et oublier. Mais plus encore : l'athéisme dont les Canadiens français se font désormais les hérauts, bien loin de se limiter à la fin de la croyance en Dieu, se traduit par une telle " vague d'incrédulité » qu'on peut prédire que bientôt ils cesseront de « croire tout court» en quoi que ce soit ${ }^{40}$. Au bout de cet athéisme se profile donc, selon Leclerc, une forme de scepticisme radical. Cet être à qui on "a enseigné que la vérité se trouvait à Rome et Rome seule », comment pourrait-il « éprouver », maintenant que «Rome l'indiffère », du " goût pour quelque vérité que ce soit ${ }^{41}$ " ? Cet être qui ne croit " guère plus en [lui]-mêm[e] qu'en $\mathrm{Dieu}^{42}$ » en vient logiquement à déduire que toutes les valeurs se valent, plus précisément à déduire qu'elles n'ont que la valeur qu'on décide de leur accorder et qu'en conséquence le plus noble et le plus vil peuvent s'échanger voire se confondre. Au peuple canadien-français il arrive ainsi « ce qui est arrivé et arrivera toujours à tous les autres peuples de même ferment allégorique [:] il se dévêt [...] [et] [...] se soulage avec la même suprême indifférence que l'on emploie à peler un fruit ${ }^{43}$ ». L'Église catholique, ayant elle-même profané les valeurs les plus hautes en les instrumentalisant, accrédite de cette façon la thèse que le réel se réduit à un simple composé de rapports entre forces, de telle sorte que les idéaux et les discours n'ont de sens ou de validité que dans la mesure où ils sont utiles à l'accumulation ou à la conservation du quantum de 
puissance dont chaque force dispose. Le régime théologicopolitique a donc engendré ce nouveau monstre : un être soidisant catholique qui en réalité non seulement hait passionnément l'Église mais embrasse en outre sans restriction aucune une forme "grossière » de "matérialisme » et d'utilitarisme ${ }^{44}$.

$\mathrm{Au}$ terme de l'évolution du régime théologico-politique se profile en définitive selon Leclerc un être parfaitement adapté à un monde plat, horizontal, sans transcendance aucune, un monde où ne circulent et ne s'échangent inlassablement que des forces, des puissances et des pseudo-valeurs (dans la mesure où celles-ci sont considérées plus ou moins utiles aux premières). Ce monde a un nom, selon Leclerc, qu'on a déjà mentionné ci-dessus : c'est l'Amérique, plus précisément l'Amérique du Nord capitaliste. L'hypothèse à première vue invraisemblable que selon Leclerc on doit pourtant envisager est que le régime ethno-théologico-politique a activement modelé une société, le Canada français, de telle manière qu'elle "passer[a] à l'histoire comme ayant été » seulement un « intermède entre sa conquête par la soldatesque britannique du XVIII ${ }^{e}$ siècle et son occupation prochaine par l'infanterie américaine du $\mathrm{XX}^{\mathrm{e} 45} \%$. Au terme de l'évolution du régime ethno-théologico-politique, pas de saints, pas de mystiques et pas même quelques grenouilles de bénitier : seulement une " âme rabougrie » qui n'aura de cesse de militer activement pour «l'intégration panaméricaine », pour l'intégration sans regret à l'« ultra-ludi[sme] de la civilisation technocratique nord-américaine " au sein duquel pourra s'épanouir librement son matérialisme grossier et son utilitarisme ${ }^{46}$. N'est-ce pas le sort des " peuples élus » que, souvent "sans variations de rituel » et sans " plissement de cœur », ils aillent « de la messe à l'hippodrome, de saint Paul à Tarzan, de la fascination de Dieu à celle du Veau d'or ${ }^{47}$ »? 


\section{Démocratie, prométhéisme et nibilisme}

Cet avenir est d'autant plus inéluctable pour le Canada français que, selon Leclerc, deux autres forces y concourent puissamment, qui sont à la fois une expression du matérialisme et de l'utilitarisme et œuvrent à son épanouissement : la démocratie et le prométhéisme.

Le matérialisme et l'utilitarisme sont d'abord indissociables de la démocratie. La lutte menée contre le régime ethnothéologico-politique mobilise en effet activement le vocabulaire et les principes démocratiques : le pouvoir parait d'autant plus injustement partagé entre un gouvernement et une Église catholique corrompus qu'on lui oppose celui que devrait exercer le peuple. Or, insiste Leclerc, la démocratie ne veut rien dire si elle ne signifie pas la consécration de la dignité du peuple et des individus tels qu'ils sont. En effet, d'abord au nom de leur droit à décider eux-mêmes de ce qu'ils veulent être ${ }^{48}$, ensuite au nom de l'égalitét9 — qui donc aurait le droit de leur commander? - il est illégitime dans un contexte démocratique de soumettre le peuple ou les individus à l'exigence de se dépasser vers une forme de transcendance ${ }^{50}$. En ce sens, la démocratie est un terreau particulièrement favorable pour le scepticisme et le relativisme : chacun y étant consacré dans son droit à être ce qu'il est ou ce qu'il veut être, la valeur de tout ce qui existe dans le monde apparait entièrement déterminée par lui. Du régime ethno-théologico-politique fondé en principe sur des valeurs transcendantes associées à la " race » française et à l'héritage catholique, le passage s'opère ainsi à une société éclatée, de plus en plus incapable de déterminer ce qui pourrait être partagé en commun. Ce passage entraine, selon Leclerc, l'émergence une société où ce qui est partagé est seulement le "plus petit dénominateur commun mental et moral ${ }^{51}$ ", c'est-à-dire les passions les plus élémentaires de l'« homme de la masse ", de l'homme de la "plèbe » ou de la «populace»: 
posséder, consommer, jouir ${ }^{52}$. En réaction à la liberté de choisir entre le Bien et le Mal dont ne cessait de les entretenir le régime ethno-théologico-politique, les citoyens démocratiques réduisent la liberté à " la faculté de choisir entre deux biens", confondant ainsi la liberté avec la " licence ${ }^{53} »$. Contre tous ceux qui pourraient espérer dans les vertus démocratiques enfin épanouies après la fin du régime ethno-théologico-politique, Leclerc reprend donc cette thèse ancienne, exposée notamment par Aristote, suivant laquelle le despotisme avilit non seulement les gouvernants, mais également les gouvernés, de telle sorte que la liberté qui lui succède est elle aussi marquée du sceau de la corruption : «Le Québec devient maitre de ses propres moyens de corruption, c'est un adulte ${ }^{54}$ !»

On le conçoit, la démocratie ainsi définie ne peut que s'associer à ce que Leclerc nomme le "prométhéisme ». Par là, il faut entendre l'épanouissement du " génie technique ${ }^{55}$ » qui vise à tout réduire à l'état de matériaux disponibles pour une humanité déterminée essentiellement par ses passions et ses désirs. Le prométhéisme consacre l'humanité comme maitresse du monde : il dispose en son centre «l'homme qui croit que la civilisation lui est due au même titre que l'intégrité de son corps et de l'air qu'il respire, et qui la juge tout aussi naturelle que ses dents et ses orteils ${ }^{56} \%$. En même temps cependant, paradoxalement, il la dépossède ou l'aliène en légitimant la « dictature œcuménique de l'Argent ${ }^{57}$ », tout ne pouvant être rendu disponible à l'humanité qu'à condition d'être réduit à l'état de marchandises. Malgré ce paradoxe ou cette contradiction, l'alliance entre la démocratie et le prométhéisme est redoutable en ce qu'elle sanctifie une certaine figure, celle de l'homme satisfait, "bien nourri et bien pourvu ", qui ne conçoit même plus la nécessité du dépassement ${ }^{58}$. En ce sens, le prométhéisme, en donnant consistance au moins en partie au vieux rêve de l'humanité de se faire l'égale de la divinité, qu'on trouve exprimé 
dès le commencement d'après la Genèse, permet d'envisager à la fois un homme accompli, baignant dans un " climat de plénitude ${ }^{59} »$ - car que pourrait-il y avoir au-delà de la satisfaction de ses passions et de ses désirs élémentaires, dont on a enfin reconnu le bien fondé ? - et un monde conçu tout entier pour lui, par là à la fois « décréé », c'est-à-dire arraché des mains du Créateur, et « recréé » par les soins de l'humanité ellemême, en fonction de ses passions et désirs ${ }^{60}$. Tout est ainsi en place pour que naisse et s'épanouisse une sorte d'« enfer ", où se réalisera l'« existence mécanique et heureuse » de "citoyens désormais américains » et qui ne concevront plus qu'on puisse être autre chose ${ }^{61}$.

C'est cette réalité que Leclerc associe au « nihilisme ${ }^{62}$ ». Cette notion doit être entendue en un double sens. D'abord, l'être athée au sens défini précédemment est porteur d'une volonté de ne plus exister en tant qu'être qui tend vers une forme de transcendance ${ }^{63}$. Par là, selon Leclerc, c'est toute une dimension de la « nature » même de l'humanité, qui s'est manifestée de diverses manières depuis le début des temps, qui se trouve niée ${ }^{64}$. Plutôt que de s'en trouver épanouie, comme le prétendent tous les faux humanistes qui se réjouissent du triomphe de la démocratie prométhéenne, l'humanité en est carrément diminuée. Mais ensuite, cet être est également porteur de la volonté de ne plus même exister comme être « rabattu » sur lui-même, sur ses passions élémentaires. Proclamé maître du monde, cet être se trouve pourtant forcément, un peu comme le «dernier homme » dont parlait Nietzsche, en réalité perpétuellement insatisfait, dans la mesure où le monde, par sa seule existence, lui oppose une résistance, ne se plie jamais entièrement à ses exigences et à ses caprices ${ }^{65}$. L'être accompli de la démocratie prométhéenne apparait ainsi tel un être épuisé, qui s'échine à réaliser l'impossible, soit l'entière soumission du réel à ses exigences, et que sa relative quoique indépassable impuis- 
sance rend à la fois fou de rage et dépressif. C'est pourquoi au bout de la démocratie ainsi entendue se profile la tentation d'en finir, le suicide. Comme le Kirilov des Possédés de Dostoïevski, l'humanité qui désire se faire l'égale de la divinité est conduite à la tentation de l'autodestruction : le «mal » de l'être démocratique et prométhéen "pourrait bien être [...] l'idée de suicide qui s'imposerait à son heure à l'âme collective ", paradoxalement " par souci de résurrection de l'homme », de son accomplissement ${ }^{66}$.

Le nihilisme dont parle Leclerc a un sens extrêmement concret. Dire que le Québec, comme on l'a mentionné, " devient maitre de ses moyens de corruption », veut dire que la "dépravation $[. .$.$] communautaire » et la corruption " géné-$ rale $^{67}$ » qui l'ont affecté risquent de miner le sens de la révolution que Leclerc sent venir très prochainement, de miner le sens de la liberté qui viendra après la fin du régime ethnothéologico-politique et qui ne sera certes pas si nouvelle qu'on voudra le croire. Ce que le Canada français ne veut pas savoir, c'est que chez lui le sens vrai de « la liberté, dont le sort est étroitement lié à celui de la vérité, s'est perdu [...] en cours de route », que «la liberté n'est plus un événement pour personne » au Québec ${ }^{68}$. À l'aube de la Révolution tranquille, Leclerc ne croit ni en l'avènement d'une société libérale bien réglée et encore moins en l'avènement d'un socialisme décolonisateur annonciateur de la transformation de l'humanité : il entrevoit plutôt le triomphe d'une humanité certes assoiffée de liberté, mais qui par là entend d'abord le déchaînement de ses passions élémentaires et, en conséquence, le triomphe d'une société fondée sur le matérialisme grossier, le prométhéisme, l'hédonisme - en un mot, ce qu'il nomme le " panaméricanisme ». Dans cette société, prédit Leclerc, le confort, l'insouciance, l'apparence passeront avant tout ${ }^{69}$, de telle sorte que la seule norme

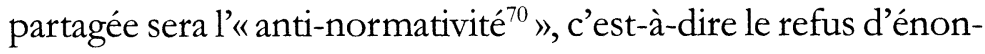


cer quelque finalité que ce soit qui paraitra brimer les passions et les désirs proclamés souverains. La société dite civile sera bien incapable, corrompue comme elle l'est, de substituer de nouvelles règles, de nouveaux rituels, de nouvelles manières collectives de faire qui pourraient se substituer aux institutions et à une normativité ancrées dans l'héritage de la « race » française et du catholicisme. Au nom de la liberté de l'individu laissé à lui-même et à ses passions, on ouvrira ainsi toute grande la porte au règne nihiliste et sans partage du monde de la marchandise et des échanges.

\section{Esprit et Histoire}

"L'existence des Canadiens français », écrit Leclerc, « a confirmé les grandes lois de l'Histoire mais elle en a également posé toutes les mêmes interrogations absolues ${ }^{71}$ ». On aurait tort en ce sens, malgré ses traits spécifiques, de chercher à comprendre l'évolution du Canada français comme si celui-ci représentait un cas unique au monde. L'analyse du Canada français que propose Leclerc s'appuie sur une philosophie de l'histoire (en même temps qu'elle la nourrit), elle-même étayée sur une anthropologie.

Pour l'humanité, tout se joue au niveau des relations qui s'établissent entre ce que Leclerc nomme l'« Esprit » et l'« Histoire ». Entre ces deux termes, point de confusion ou de recoupement (comme chez Hegel par exemple) dans la mesure où l'Esprit désigne un lieu qui déborde l'Histoire. En même temps, cependant, l'Esprit ne peut pas se manifester ailleurs que dans l'Histoire, de telle sorte que, devant constamment frayer avec ce qui le nie, il est fragile et est même constamment menacé de disparaître. L'enjeu, selon Leclerc, est donc de trouver des moyens permettant à l'Esprit de se situer au dedans de l'Histoire mais de telle sorte qu'il soit simultanément en dehors d'elle, voire dressé contre elle. 


\section{L'anthropologie : l'Esprit}

Qu'est-ce que l'Esprit? Plutôt que d'en donner une définition en bonne et due forme, Leclerc procède en circonscrivant patiemment cette notion, donnant au lecteur, par touches successives, différents aperçus de ce qu'elle est. Seule manière, peut-on penser, de parler du «mystère » qu'elle recouvre ou de l'« infini » auquel elle renvoie ${ }^{72}$. Tâchons donc de le suivre dans son effort de circonscrire sans définir, en relevant l'un après l'autre les principaux éléments qu'il associe à l'Esprit : la « nature », la « liberté », l'« autorité », la " grâce », la « culture », la "personne ».

L'Esprit entretient d'abord un rapport étroit avec la « nature $^{73} »$. Il faut admettre, selon Leclerc, qu'il existe une chose telle que des « lois naturelles » ou des « lois de la nature humaine $»^{74}$. La nature ici n'est pas à entendre au sens des Modernes, c'est-à-dire comme un déterminisme, mais plutôt au sens des auteurs classiques et chrétiens, c'est-à-dire comme un telos vers quoi tend l'être humain ${ }^{75}$. Ce telos est précisément l'Esprit, ce qui permet de parler d'une " angoisse originelle de tout homme » qui le pousse à une " évolution spirituelle ", dont il faut chercher à prendre la mesure ${ }^{76}$. Cette évolution suppose des médiations, dont la description doit permettre de circonscrire plus précisément l'Esprit, au-delà de cette première esquisse de définition : l'Esprit est ce vers quoi tend l'humanité quand elle se conforme à sa finalité naturelle.

Puisque la nature entendue au sens d'une finalité n'est pas un déterminisme, elle n'est pas antinomique avec la « liberté », qui est la première de ces médiations. C'est, autrement dit, librement que l'humanité peut se plier à sa finalité naturelle, peut s'orienter vers l'Esprit. C'est pourquoi la liberté peut être définie comme l'« application » de la « loi naturelle ${ }^{77}$ »; c'est aussi pourquoi il est interdit de la confondre avec la « licence », qui récuse toute orientation ou finalité ${ }^{78}$. La licence est 
nécessairement une escroquerie, "un mythe des plus cruels ", puisque proclamant en principe l'humanité maitresse d'ellemême et de tout le réel, elle l'asservit en réalité à la fois à ses passions et aux moyens de les apaiser, à l'« argent » et à ses " normes de vulgarité ${ }^{79}$ ». Mais comment dès lors rendre compte de l'attrait qu'exerce la licence ? C'est que la nature orientant la liberté sans la déterminer, l'humanité demeure au fond toujours libre de réaliser ou non sa nature ${ }^{80}$. L'enseignement du christianisme - c'est là le sens profond du dogme du " péché originel ${ }^{81} »-$ est d'ailleurs que la liberté humaine tend dès le commencement à échapper à sa nature ou à sa finalité naturelle, à se confondre avec la licence.

Cette confusion originelle de la liberté humaine avec la licence et avec la désobéissance est au même moment, paradoxalement, l'acte de naissance de l'« autorité ». C'est précisément parce que l'humanité s'est éloignée dès le commencement de sa nature que l'autorité est née afin de la contraindre à la réaliser, c'est-à-dire à s'orienter vers l'Esprit. L'autorité n'a de sens, historiquement, que d'« appliquer ses facultés et énergies à corriger la bête à péché originel ${ }^{82} »$. Aussi n'est-ce pas un hasard si l'autorité est dans un premier temps d'essence religieuse : " [1] pouvoir a, dès le début de l'embryon social, été un sacerdoce » et a ainsi été nimbé d'un " halo [...] d'effroi ${ }^{83}$ ». Le régime théologico-politique, en ce sens, loin d'être une aberration (ou de tenir seulement à des circonstances historiques particulières) a des assises profondes dans la façon même dont l'humanité use de sa liberté dès le commencement.

Cela n'empêche pas que tout régime théologico-politique se trouve enferré dans des contradictions qui le minent inéluctablement. Visant à orienter l'humanité vers la finalité naturelle dont elle s'est éloignée, un tel régime cherche inévitablement à réaliser cet objectif en circonscrivant la liberté humaine. Autrement dit, le régime théologico-politique est tou- 
jours négateur de ce qu'il cherche en principe à restaurer dans la mesure où, au nom de la finalité naturelle dont s'est éloignée l'humanité, il limite la liberté humaine, qui est pourtant la voie princière par laquelle cette finalité peut être atteinte. Concrètement, cela signifie que l'autorité d'essence théologico-politique revêt les traits d'un puissant appareil de contrôle et de contrainte des êtres humains. En outre, cet appareil tend forcément à user, afin d'orienter à nouveau ceux-ci vers l'Esprit, d'un langage et de représentations qu'ils peuvent aisément comprendre, c'est-à-dire qui font appel à une imagerie ou à une symbolique particulières, ancrées dans des traditions, des contextes particuliers. Ces représentations ne pouvant jamais être présentées pour ce qu'elles sont en réalité, soit des produits contingents, historiques, par là susceptibles d'êtres interrogés, discutés et contestés, elles sont donc présentées pour ce qu'elles ne sont pas, soit des absolus indiscutables et incontestables (en ce sens, le régime théologico-politique s'appuie, par définition, sur le mensonge). Ces représentations particulières ne " vien[nent] pas compléter la nature, mais bien s'y substituer la plupart du temps ${ }^{84}$ ». Comme cette substitution finit tôt ou tard par être révélée, ne serait-ce que parce que les représentations de l'Esprit apparaissent différentes et souvent contradictoires d'une société à l'autre ou d'une époque à l'autre, il faut conclure que, dans les faits et à son corps défendant, le régime théologico-politique tend à accréditer l'idée que l'Esprit n'a pas d'autre consistance que l'ensemble des représentations qu'on en donne, en somme qu'il n'a pas de réalité propre. Au bout d'un certain temps, il semble donc que l'appareil de contrôle et de contrainte des êtres ne s'appuie plus que sur « la mythologie visible de ce qui fût [sic] naguère une métaphysique ${ }^{85} \gg$. Pour les êtres soumis au régime théologico-politique et qui découvrent la tromperie sur lequel il repose, deux attitudes sont dès lors envisageables : ou bien se plier formellement aux règles du 
régime à des fins qui leur sont propres, de telle sorte que l'autorité ne sera respectée qu'en autant qu'elle s'accorde avec l'« utilité ${ }^{86}$ » ou avec la recherche de la satisfaction des passions ; ou bien rompre avec ce régime hypocrite et avec toute l'imagerie des finalités naturelles au profit de l'établissement d'un nouveau régime fondé sur la liberté confondue avec la licence. En somme, comme l'avait déjà compris Platon, le régime théologico-politique est une impossibilité ou ne peut que se nier en se réalisant (c'est pourquoi l'autorité est « le commencement de rien ${ }^{87}$ ») : "Il n'y aurait qu'une méthode irréfutable de prouver que l'autorité vient de Dieu et ce serait la sainteté $[\ldots]$ ou, tout au moins, la sorte de sagesse surhumaine préconisée par Platon et déjà reconnue chimérique par lui ${ }^{88}$. » De l'autorité théologico-politique il ne reste ainsi bientôt plus que le principe de l'autorité nue, considérée utile aux êtres en autant qu'ils poursuivent des fins privées ou particulières : ainsi, tout se passe comme si les "prêtres ", ces " admirables pionniers des peuples, des civilisations et des cultures », et les « castes cléricales ", ces " géniales organisatrices », n'avaient rien fait d'autre que poser les fondements de la réalité purement temporelle qu'est l'État ${ }^{89}$.

Contre la fatale dégradation du régime théologico-politique et contre l'utilitarisme qu'il fait triompher il n'y a plus dès lors qu'un seul recours : la " grâce ${ }^{90}$ ». Celle-ci est la deuxième médiation, après la liberté. L'insuffisance de celle-ci rend nécessaire la grâce pour atteindre l'Esprit. Si la liberté est naturelle, donc à la portée de tous, la grâce n'est en revanche offerte qu'à quelques-uns et acceptée par peu' ${ }^{91}$. Tout se passe comme si, après la Chute, la nature ou la liberté ne pouvait s'accomplir que par le recours à la surnature. La grâce consiste en ce que l'Esprit (pneuma) souffle là où il l'entend, « où bon lui semble », c'est-à-dire qu'il s'impose " comme un démon intérieur » au petit nombre qui est ainsi appelé à vivre sa liberté en 
réalisant les finalités naturelles envers et contre tout et notamment contre le grand nombre qui le méprise et tend à l'utilitarisme ${ }^{92}$. En ce sens, la grâce seule permet de contrer l'« antihumanisme $e^{93}$ " qu'engendre le régime théologico-politique, c'est-à-dire d'en arriver à un rapport de certains êtres humains à eux-mêmes fondé à la fois sur la liberté et la réalisation de leur nature.

De ce qui précède découle la thèse que l'Esprit ne revêt pas d'autre forme que la liberté humaine réalisant, par grâce, sa nature : l'« Esprit défaille quand la liberté ne veille et ne prie $^{94}$ », puisqu'il n'est rien d'autre que « la plus haute forme de la Vie sur terre ${ }^{95}$ \%. Poussant la logique de son propos jusqu'au bout, Leclerc écrit qu'à l'athéisme entendu comme utilitarisme ou matérialisme s'oppose ainsi une autre forme d'athéisme, cette fois au sens d'un refus de considérer et de présentifier l'Esprit comme une entité à part et à distance de l'humanité ${ }^{96}$ : l'Esprit est l'humanité en tant que celle-ci se réalise pleinement (du moins en quelques-uns de ses représentants, touchés par la grâce). Cet athéisme, paradoxalement, Dieu le veut, et c'est même là le sens de la Création de l'humanité : si les (faux) prophètes proclament " que l'Histoire n'obéit qu'à la volonté des hommes, en définitive, la leur ", c'est afin d'empêcher "qu'elle soit ce que Dieu veut qu'elle soit, [c'est-à-dire] une odyssée de la liberté ${ }^{97}$ » humaine. Il a fallu le régime théologicopolitique ou le " dépouill[ement] » de la " sensibilité surnaturelle » de l'être humain " au nom du Dieu dominical " pour faire oublier que le christianisme considéré en son essence, c'està-dire du point de vue du mystère de la Trinité par quoi l'Esprit relève le Fils, est " une forme suprême d'humanisme " ». Leclerc va ainsi jusqu'à écrire que c'est seulement de l'être humain pleinement et véritablement libre que l'on peut espérer retrouver Dieu, « retourner ${ }^{99}$ » à lui : seule la corruption induite par le régime théologico-politique a pu faire oublier « [q] ’’il est 
inutile, vain et malhonnête de vouloir sauter l'étape fondamentale de l'humanisme, ou perfectionnement de l'homme dans $l^{\prime}$ homme ${ }^{100}$ " pour atteindre Dieu.

L'humanisme ou la liberté humaine réalisant sa nature par grâce se reconnait à des manifestations privilégiées. La « culture » entendue comme capacité de " création ${ }^{101}$ » ou comme "po-é-ti-que ${ }^{102}$ » en est la principale : la « liberté, [...] esprit-né, s'exprime dans la qualité de création ${ }^{103} »$. C'est dans l'œuvre d'art, en particulier - mais aussi, il est vrai, dans l'œuvre philosophique ou scientifique, - que s'exprime le " côté sacral » ou l'« élan spirituel » de la " pensée ${ }^{104} »$. L'artiste (et non le divertisseur bien sûr) exprime des " essences spirituelles ${ }^{105}$ ", qui se reconnaissent à ce qu'elles « imitent » (au sens de « recommencent ») la Création divine en donnant naissance à des œuvres qui durent dans le temps, qui cherchent à se doter d'une dimension universelle ${ }^{106}$. Que le régime théologico-politique haïsse autant l'art libre et créateur n'est donc nullement un hasard, car cet art, qui manifeste l'Esprit, s'oppose en tout point à l'art instrumental et édifiant que les autorités tolèrent ou encouragent parce qu'elles le jugent utile.

Cependant, s'il y a bien une "substance divine en l'homme ${ }^{107}$ » manifeste dans ses capacités de se faire créateur ou artiste, l'humanité, insiste Leclerc, ne peut jamais se confondre purement et simplement avec la divinité. Comme l'enseigne ici encore le récit judaïque et chrétien du commencement, l'humanité ne naît pas autrement que d'une séparation avec le divin, de telle sorte que la divinité n'a de sens pour elle que d'être perdue ou «abolie ${ }^{108}$ ». Si la culture ou l'art sont des manifestations de la liberté humaine, donc de l'Esprit, ils prennent pourtant la suite du " dépouillement de l'Arbre de la Science, du Bien et du Mal » et se situent à «l'inverse même de l'état adamique ${ }^{109} »$. L'humanité, autrement dit, est paradoxalement à la fois le lieu où se manifeste l'Esprit et une entité prise 
dans les rets de la finitude ${ }^{110}$. L'humanité est constituée d'individus sensibles, souffrants et mortels, par là situés dans un rapport à la perfection divine qui la leur révèle inaccessible en sa plénitude, comme le rappelle d'ailleurs la personne du Père à jamais invisible et absente (par contraste avec la personne du Fils) dans le christianisme. C'est pourquoi l'odyssée humaine de la liberté peut se lire comme un effort à jamais inachevé visant la "rédemption ${ }^{111}$ » : la pensée ou l'intelligence, quand elle vise à faire de la terre le lieu de l'Esprit, " travaille» (pour parler comme Baudelaire cette fois) à l'impossible « disparition des traces du péché originel ${ }^{112} »$. Dit autrement, toute création humaine repose sur l'inachevable effort de la chair pour rejoindre le divin, pour se faire " chair abolie ${ }^{113}$ ».

Tout ceci signifie en définitive que l'être humain est indépassablement un être historique même s'il veut constamment, du moins quand il est élevé par la grâce, se délivrer « de la fosse aux lions de l'histoire ${ }^{114}$ ». L'humanité, en ce sens, est située sur deux plans qui sont à la fois indissociables et antinomiques : " La culture est la perpendiculaire $C D$ que l'intelligence humaine doit élever sur une ligne horizontale $A B$ qui se nomme l'Histoire et lui sert de tremplin ${ }^{115}$. " C'est pourquoi les manifestations de l'Esprit n'existent jamais à l'état pur : l'art et la culture sont toujours situés dans le temps et dans l'espace, ils sont toujours historiques même s'ils visent à transcender l'Histoire pour se rapprocher de ('inatteignable) rédemption et de Dieu. Ainsi peut-on parler, par exemple, d'un " esprit français ", qui est une " semence spirituelle dans un secteur précis du globe ${ }^{116} »$. S'il faut œuvrer à préserver un tel esprit, qui fait advenir mais toujours dans l'incomplétude des dimensions de la "spiritualité ", c'est afin d'entretenir avec lui des rapports fondés sur le dialogue afin d'éviter qu'il ne se dessèche et afin de le faire se manifester de nouveau et autrement, dans les contextes où il a migré ${ }^{117}$. Cet effort de reprise des « essences 
spirituelles » pour les enrichir est l'œuvre de ce que Leclerc nomme l'« âme ${ }^{118}$ », dont la manifestation en l'individu fait de celui-ci une "personne », laquelle désigne "la portion de l'homme susceptible d'éternité ${ }^{119} »$.

Or, diagnostique Leclerc, notre âme est « rabougrie ${ }^{120}$ ", notre monde se «dépersonnalis[e] ${ }^{121}$ » et souffre en conséquence d'une « usure spirituelle $e^{122} »$. Pour le dire autrement : l'Histoire se vide de l'Esprit ou l'Esprit se perd dans l'Histoire plutôt que de s'y réaliser. Pourquoi ?

\section{La philosophie de l'Histoire : l'Esprit, le Bien, le Mal}

Si Leclerc fait de l'Histoire le seul lieu de manifestation ou de réalisation de l'Esprit, il refuse cependant, comme on l'a dit, d'identifier l'une à l'autre et insiste plutôt sur leur opposition irréductible.

Pour mieux comprendre ces rapports d'opposition, il faut d'abord se rappeler que l'Histoire et l'Esprit ont une commune origine. Tout commence, pour Leclerc, avec la Chute ou le péché originel. Par là, il faut entendre que tout commence avec la séparation de l'humanité d'avec la divinité, que tout commence avec l'absence, pour l'humanité, de Dieu ou de la loi divine en ce monde : bien que tout ce qui est soit par définition une Création divine, du point de vue des êtres humains on ne peut rien faire remonter directement à Dieu, l'« autorité ne vient pas plus de [Lui] que la raison, la flore ou le système solaire ${ }^{123}$ ». S'il y a établissement d'un régime théologico-politique, dont on a vu qu'il conduisait à l'utilitarisme et au matérialisme par ses contradictions mêmes, s'il y a, autrement dit, quelque chose comme une Histoire, et s'il y a, par les médiations de la liberté ou de la grâce, manifestations de l'Esprit, c'est dans les deux cas en réaction à cette absence de Dieu dans le monde. Cependant, cette commune origine ne doit pas faire perdre de vue la différence qui sépare l'Histoire et l'Esprit. 
L'Histoire découle à l'origine du désir de combler l'absence de Dieu sur terre ou d'incarner positivement le Bien en instituant une autorité dont le fondement est théologique. Comme on l'a $\mathrm{vu}$, les contradictions de ce type de régime induisent fatalement à l'athéisme entendu comme négation de Dieu - autrement dit, au Mal, qui, précise Leclerc, « existe » ainsi « en soi ${ }^{24}$ ». L'Esprit, de son côté, au contraire, consiste plutôt à simplement rappeler l'absence de Dieu sur terre et ainsi à manifester, au travers de la création ou de l'art, qui sont à jamais inachevés et inachevables, l'absence du Bien dans sa plénitude pour nous. Certes, l'objet est ici également de manifester l'« absolu », mais comme le temps suppose sa nécessaire " adaptation ${ }^{125}$ " aux circonstances, celui-ci est énoncé à la fois comme présent et absent dans la création ou l'art, ce qui préserve les chances de la liberté et de nouvelles manifestations de l'Esprit. Si quelque chose comme le Bien existe dans les sociétés humaines, c'est donc en tant qu'il est à venir ou qu'il se manifeste comme un horizon seulement.

Ce qui fait de la tradition judéo-chrétienne un extraordinaire révélateur de notre condition, c'est qu'elle permet d'énoncer selon Leclerc ces deux positions à la fois, qu'elle est au fondement tant de l'Histoire que de l'Esprit, tant du régime théologico-politique et de ses suites que de la liberté, qu'elle célèbre à la fois la " chair et [l']esprit ", le " temps et ['] éternité ${ }^{126} »$. D'un côté, le judaïsme et le christianisme, à l'origine, sont l'affirmation simultanée de l'absence de Dieu pour nous et de l'impossibilité d'oublier cette absence : irreprésentable dans le judaïsme, qui interdit les idoles, le « Je suis qui je suis " de l'Exode s'incarne, dans le christianisme, dans la personne du Fils, mais pour être finalement crucifié et ainsi abandonner les êtres humains à eux-mêmes, à leur liberté, à l'Esprit qui dépend autant d'eux que de la grâce. En même temps, de l'autre côté, le judaïsme et le christianisme sont tout 
aussi bien fondés sur la volonté de présentifier Dieu et de réaliser le Bien sur terre : le Dieu irreprésentable de l'Exode a pourtant sa Loi, son peuple élu ou sa nation sainte dans le judaïsme, son Église censée être son vicaire sur terre et son complément théologico-politique dans le christianisme - avec, à chaque fois, au terme de l'évolution de ces institutions, l'utilitarisme et le matérialisme, le triomphe du monde temporel ou de l'Histoire.

Ni pour le judaïsme ni pour le christianisme considérés globalement il n'y a contradiction entre ces deux dimensions : on doit constater que les « peuples élus [...] ne croient guère au $\mathrm{Mal}^{127} \gg$. Pis encore, même quand ils en viennent à apercevoir et à admettre l'existence du Mal, c'est pour mieux l'inscrire dans une " dialectique ${ }^{128}$ » qui promet son dépassement dans une synthèse supérieure : c'est le travail de la philosophie, audelà de la théologie, que de " consoler » les peuples en leur " conseillant de fermer les yeux », en cherchant à les " convainc[re] que les vices sont toujours provisoires, interchangeables et promis à la durée des roses ${ }^{129} »$. Cette dialectique, qui reconnait le $\mathrm{Mal}$ pour en faire un moment de la réalisation du Bien, qui reconnait, autrement dit, les maux de l'Histoire pour les confondre avec la réalisation de l'Esprit, marque en fait le suprême triomphe de l'Histoire et du Mal, puisqu'elle consacre indifféremment tous les événements, passés, présents et à venir en les inscrivant dans une trame d'ensemble qui leur donne sens et les " rachète ». Le Mal, quand il est ramené à un moment du Bien, disparaît donc : "Aussi longtemps que nous pouvons nier l'existence du mal et nous berner de l'illusion qu'à la fin des fins le bien triomphera, nous le faisons sans scrupule. Un seul détail nous échappe : le mal existe en soi ; nous sommes ses meilleurs propagandistes et ses plus féconds terrains d'exercice ${ }^{1.30} »$. 
Selon Leclerc, les sociétés contemporaines vivent précisément de cette croyance que tous les maux apparents ne sont que des moments ou des étapes de la réalisation du Bien. La guerre n'est qu'un moment qui prépare la paix ou la sécurité et le confort ${ }^{131}$; l'apparent aplatissement de l'humanité sur la vulgarité des passions et des instincts que suppose la démocratie libérale promothéiste et hédoniste ou même l'apparente inhumanité d'une institution comme le marché qui consacre le règne aliénant de l'argent, ne sont que des moments qui préparent le règne d'une humanité accomplie, satisfaite ${ }^{132}$. On a pu penser que l'humanité se distinguait non seulement de la divinité mais aussi de l'animalité, qui ignore la distinction entre le Bien et le Mal : c'est, tout au contraire, en faisant disparaître la " marge friable » qui la sépare de l'animalité, c'est-à-dire en s'identifiant à cette dernière par le refus de distinguer entre le Bien et le Mal et en suivant aveuglément ses passions, que l'humanité prétend s'accomplir pleinement ou atteindre à la divinitét $^{133}$.

En ce sens, l'Histoire prétend accomplir la rédemption de l'humanité et avec elle l'« expiation » de tous ses crimes ou péchés ${ }^{134}$. Il en résulte un état d'« euphorie constante ${ }^{135}$ » dans lequel baignent des êtres qui s'efforcent d'oublier la souffrance, qui vivent sans penser à rien d'autre que la jouissance ou la consommation, présente ou à venir. Parmi ces êtres, il n'y a littéralement " personne », il n'y a pas de "personnes » au sens où l'entend Leclerc. En ce sens, l'Histoire achevée, qui prétend correspondre au réel en sa totalité, est la somme et le résultat final de toutes les " amputations spirituelles ${ }^{136}$ » qui ont précédé.

Mais peut-on dire pour autant que l'Histoire ou le Mal triomphe sans reste ? Il arrive bien à Leclerc de croire que « la partie de cartes [...] paraît perdue ${ }^{137}$ ». Cependant, en rester là serait oublier la grâce. Contre ou plutôt " malgré la société ${ }^{138}$ ", 
le petit nombre persiste à faire se manifester l'Esprit. Le poète, par exemple, qui refuse de croire que tout est accompli, rappelle l'impossible éradication de tout le Mal en montrant que la « souffrance n'est pas une absurdité ${ }^{139}$ » et qu'elle fait plutôt partie de la condition humaine. À ses semblables, il n'a de cesse d'enseigner rudement l'inaccomplissement : «Irréconciliable à lui-même parce qu'il a une conscience aiguë de la finitude du temps, de la chair et de la futilité de l'orgueil, il ne veut pas se reposer avant que l'unité de la chair et de l'esprit soit, par lui, accomplie et tant qu'il restera une parcelle de chair à tuer dans l'esprit ${ }^{140} »$. Le poète, comme tout autre créateur ou ami des " essences spirituelles », appartient à une sorte de " tiers-état ${ }^{141}$ » des sociétés promothéistes et hédonistes, porteur d'une promesse de révolution au sens personnaliste, c'est-à-dire de l'institution d'une communauté d'êtres qui habitent le monde autrement que les citoyens satisfaits en témoignant de la grâce : le "poète et son œuvre ne viennent pas du milieu social ou de l'habitat professionnel; ils viennent d'ailleurs, de bien plus loin encore ; ils viennent de là où les chiffres terrestres n'ont jamais eu accès. Ils viennent de l'au-delà de l'intelligence et du cœur. Pourquoi, après tout, ne viendraient-ils pas de $\mathrm{Dieu}^{142}$ ? "

Dans le contexte nord-américain, cette sorte d'irruption toujours plus ou moins imprévisible d'une communauté de " personnes » peut revêtir deux formes en particulier selon Leclerc. Celui-ci, d'abord, envisage qu'elle puisse prendre une forme politique : contre l'« absurdité spirituelle du Canada ${ }^{14.3}$ ", qui est par excellence le pays de l'Histoire sans Esprit, de la satisfaction béate induite par le promothéisme et l'hédonisme, il se peut que se dresse un mouvement pour qui l'« enjeu souverain de l'indépendance politique du Québec [...] [sera] l'esprit humain, son acquittement et sa réhabilitation sans condition $^{144}$ ». Cependant, les inévitables compromissions auxquelles devra se plier le mouvement « séparatiste », dans la mesure 
où il devra se soumettre aux règles du jeu politiques et chercher à gagner une majorité de citoyens, rendent en définitive Leclerc des plus sceptiques quant à ses capacités à manifester l'Esprit : tout donne en fait à penser que l'« acte de sécession serait un geste de nature politique » et qu'en conséquence « il ne résoudrait nullement le problème culturel du Québec français et combien moins encore les angoisses métaphysiques du Canada français! Le séparatisme ne chargera le Québec d'aucune énergie spirituelle supplémentaire ${ }^{145} \%$. Aussi, c'est plutôt un "schisme intellectuel ${ }^{146}$ " que favorise Leclerc, qui permettrait à une communauté de " personnes » de se déployer en quelque sorte parallèlement à la majorité des citoyens et à l'autorité établie. L'autorité, fondée sur l'utilitarisme et le matérialisme, ne peut que continuer de s'exercer et Leclerc n'en propose en définitive nullement la remise en question : "[i]l n'existe aucune opposition entre l'autorité et la liberté, car la première est d'ordre essentiellement pratique et la seconde est une essence spirituelle. Le contraire de l'autorité n'est pas la liberté mais une autre autoritéé ${ }^{147} »$. Avec l'autorité, il importe que l'Esprit puisse en fait conclure une sorte de pacte de nonagression : si l'autorité cesse d'exercer la censure contre lui, il ne la contestera pas, de sorte que nous en « venions enfin à la division du travail, ce principe fondamental d'efficacité, de valeur et de perfection en matière d'économie comme de cul$\operatorname{ture}^{148} »$. Au petit nombre, aux " personnes » qui veulent l'Esprit, il faut un espace de liberté ; à la majorité, aux citoyens qui le refusent et qui veulent le promothéisme et l'hédonisme, il ne faut que l'autorité nécessaire à l'élaboration de règles minimales qui permettent les échanges et la prospérité. À la limite, la posture proposée par Leclerc aboutit à une quasi-défense du statu quo: il « est logique que la société, toute société [...] et que l'autorité qui en est la clé de voûte, se vouent à l'idéal de l'efficacité technique ${ }^{149}$ » et il absurde de penser qu'il pourrait en 
être autrement, qu'une réforme de la société dans son ensemble est possible. Il faut ménager un espace à la liberté mais après avoir admis qu'elle n'a "pas d'ambition politique et commerciale $^{150} »$.

\section{$* * *$}

On comprend mieux au terme de cette analyse que la réception de l'œuvre de Gilles Leclerc n'ait pas eu lieu il y a quarante ans. Pour ses contemporains, cette œuvre pouvait paraittre fondée sur des paradoxes dont ils ne savaient trop que faire. D'un côté, Leclerc attaquait avec une férocité rarement égalée les fondements mêmes du régime ethno-théologicopolitique et il annonçait son déclin inéluctable : en même temps, de l'autre, il proposait un personnalisme qui puisait largement dans l'héritage de la philosophie classique et du christianisme tout en refusant l'idée d'une réforme globale de la société et en se montrant tout à fait pessimiste quant aux changements qui s'annonçaient. Comme on l'a déjà dit, la pensée de Gilles Leclerc, en ce sens, ne pouvait que se situer en marge ou à l'écart des courants qui ont participé de la gestation ou de l'accomplissement de la Révolution tranquille.

Reste à poser la question de ce que peut signifier cette œuvre pour nous maintenant, pour les contemporains que nous sommes. Peut-on la lire avec profit même si, bien sûr, la société présente n’a plus grand-chose à voir avec le régime ethnothéologico-politique qu'il décrivait ?

D'abord, on pourrait probablement s'entendre sur le fait qu'il est quand même difficile d'ignorer un auteur qui a bien été l'un des seuls non-traditionalistes, sinon le seul, à prédire que la liberté nouvelle tant célébrée au cours des années 1960 devait aboutir à une forme d'hédonisme ou de consumérisme assorti à une adhésion quasiment sans réserve au capitalisme nord-américain. D'une certaine façon, Leclerc anticipait l'échec 
des courants et des mouvements qui voulaient édifier sur la base du souffle de liberté portée par la Révolution tranquille une société qui, socialiste, social-démocrate ou libérale progressiste, se serait distinguée des autres sociétés nord-américaines. Toute réflexion qui estime que cet échec est désormais consacré et qu'on doit partir de lui pour repenser les fondements de la société québécoise ne peut, en ce sens, faire l'économie de la prise en compte d'une pensée comme celle de Gilles Leclerc (comme d'ailleurs, ainsi qu'on l'a déjà mentionné, de ces penseurs tels Dumont, Vadeboncœur, Grand'Maison ou Léon Dion qui, ayant pour leur part d'abord partagé pour un temps l'optimisme des années 1960, ont ensuite dit leur désenchantement).

Cela étant dit, on ne saurait ignorer que la critique proposée par Leclerc vise moins les structures du capitalisme nordaméricain que le mode de vie - l'«American way of life », pourrait-on dire - qui le rend possible et légitime aux yeux des citoyens. Le capitalisme nord-américain ne constitue en rien selon Leclerc une structure oppressive imposée de l'extérieur et contre lequel le peuple ne songerait qu'à se dresser ; plutôt, il correspond aux aspirations d'une société disposant en son centre une figure de la subjectivité qui, au nom de sa liberté, célèbre l'« anti-normativité ». C'est parce qu'il persistait à s'inscrire envers et contre tout dans une tradition de pensée et de réflexion qui dispose la question des finalités - ce qu'il nommait les «finalités naturelles » - en son centre, que Leclerc n'a pu qu'être extrêmement sensible à la question de la normativité et, en fait, à son déclin, qu'il a annoncé ici encore avant même qu'il ne se produise. Or si plusieurs s'inquiètent aujourd'hui au Québec de la domination apparemment sans limite du capitalisme nord-américain et cherchent ce qui l'a rendu possible, on en voit beaucoup moins accepter de lier cette domination à la figure de la subjectivité née dans la foulée des soi-disant mou- 
vements d'émancipation des années 1960. On pourrait même avoir l'impression, par exemple à la lumière du discours critique de la mondialisation en vogue, que la critique du capitalisme nord-américain s'énonce à partir et au nom même de cette figure, qui se sent brimée dans sa liberté ou aliénée par le mécanisme du marché. Tout indique qu'après avoir associé étroitement le régime théologico-politique ou l'héritage catholique aux finalités ou à la normativité, nous soyons devenus incapables, ayant rejeté les premiers, à la fois de poser la question soulevée par l'absence des secondes et de concevoir que cette absence puisse avoir quoi que ce soit à voir avec notre malaise actuel.

De la mémoire nationale québécoise, il est désormais devenu de bon ton de demander ce qu'il faudrait en conserver ou en tirer pour penser notre présent et notre avenir. Cette mémoire est interrogée, soupesée, examinée sous toutes ses coutures : avons-nous ou non été un peuple à part, ou bien encore simplement des Américains parlant français, somme toute semblables à nos voisins ? Notre société a-t-elle été si autoritaire que certains historiens l'ont prétendu ou avons-nous été à peu près aussi libéraux que tout le monde ? Etc. Cette interrogation multiforme pointe le plus souvent, à son corps défendant (sauf dans des travaux spécialisés), vers un véritable point aveugle de notre histoire et de notre mémoire : l'héritage religieux, chrétien, catholique. De celui-ci, la plupart des penseurs de notre " récit mémoriel » ne savent que faire et ne veulent rien savoir le plus souvent. Si l'œuvre de Gilles Leclerc nous est précieuse, c'est qu'elle nous rappelle que cet héritage, qu'il défend au moment même où il œuvre lui-même à abattre le régime théologico-politique, est porteur, par delà même les réponses qu'il leur apporte (certes tout à fait discutables), de questions essentielles suscitées par le vivre-ensemble : celles de la «bonne vie », des finalités, de la Loi - questions dont 
l'incapacité de les poser laisse la subjectivité à elle-même et empêche sa structuration de manière à lui permettre de se défendre contre les systèmes de domination. C'est aussi, probablement, la raison pour laquelle cette œuvre nous demeurera étrangère ou inaccessible, du moins tant que nous n'aurons pas appris à considérer et à apprécier avec plus de distance et de justesse notre passé catholique.

\section{NOTES}

${ }^{1}$ La première ébauche de cet article fut présentée sous forme de communication au colloque «Les enjeux de la citoyenneté dans les sociétés pluralistes », organisé par le Centre interdisciplinaire de recherche sur la citoyenneté et les études minoritaires (CIRCEM), tenu à Ottawa, les 9-11 novembre 2000. L'auteur tient à remercier les participants à la discussion qui a suivi pour leurs critiques et commentaires, en particulier Daniel Tanguay, Martin Meunier et Jean Pichette.

${ }^{2}$ Né le 24 juin 1928 à Saint-Rosaire d'Arthabaska, Gilles Leclerc est mort à Québec, le 27 septembre 1999 (sa mort n'ayant suscité aucune réaction du milieu intellectuel et littéraire). Il a été journaliste au journal Le Devoir et à Radio-Canada au cours des années 1950 avant de devenir, en 1963, le premier employé professionnel de l'Office de la langue française, où il a travaillé jusqu'à sa retraite, se consacrant particulièrement à une collection intitulée Néologie en marche. Il fut l'ami, notamment, d'André Major, de George Dor et de Jean Marcel.

${ }^{3}$ Gilles Leclerc, Journal d'un inquisiteur, Montréal, Éditions de l'Aube, 1960, p. 274 note 1 ; Gilles Leclerc, Journal d'un inquisiteur, Montréal, Éditions du Jour, 1974, p. 11. L'ouvrage a été réédité chez Lux, en 2003.

${ }^{4}$ Journal d'un inquisiteur (édition de 1974), op. cit., pp. 11, 13.

${ }^{5}$ André Major, « Mémoires d'une jeune Canoque. VII », L'Action nationale, vol. 55, no 8 (avril 1966), pp. 986-991. Voir également Jean-Marc Piotte, $U n$ parti pris politique. Essais, Montréal, VLB, 1979, p. 13.

"Voir notamment Camille-H. Mailhot. " Gilles Leclerc - Journal d'un inquisiteur. Montréal, Éditions de l'Aube, 1960. 313 p. ", dans Archives des Lettres canadiennes, Ottawa, Éd. de l'Université d'Ottawa, 1961, tome I, p. 335 ; 
Paul Gay, Notre roman, Montréal, HMH, 1973, p. 118 ; Pierre-Louis Vaillancourt. "Gilles Leclerc, inquisiteur », dans Paul Wyczynski, François Gallays et Sylvain Simard, dir. Archives des Lettres canadiennes: L'essai et la prose d'idées au Québec, Montréal, Fides, 1985, tome VI. Pp. 689-695.

${ }^{7}$ Dorval Brunelle, Les trois colombes, Montréal, VLB, 1985, p. 214 ; Jacques Pelletier, I e poids de l'histoire, Montréal, Nuit Blanche, 1995, p. 311.

${ }^{8}$ Jean Marcel, «Préface » dans Gilles Leclerc, Journal d'un inquisiteur (édition de 1974), op. cit., p. 27.

"Sur la pensée de droite après la Révolution tranquille, voir Xavier Gélinas. " Déclin et disparition de la droite intellectuelle québécoise (1956-1966)». Société, nos 20-21 (été 1999), pp. 95-110.

1" On pourrait peut-être la rapprocher, sans ignorer qu'elle n'a pas partagé leur optimisme originel, de ces pensées qui, après avoir beaucoup espéré de la Révolution tranquille, ont ensuite insisté sur le désenchantement qu'elle a suscité : par exemple, celles de Fernand Dumont, de Pierre Vadeboncœur, de Jacques Grand'Maison, de Léon Dion.

"Gilles Leclerc, "Prométhée ou Schweitzer ", dans Gilles Leclerc et al. Cabier pour un paysage à inventer, [s.l.], 1960, p. 14. Il faut relever le nom du «situationniste » français Guy Debord (orthographié « Debord » sur la page couverture mais «Debard » à la p. 97) au sommaire de cette publication, dont il semble n'y avoir eu qu'un seul numéro et qui a été vraisemblablement dirigée par Patrick Straham. On relève également au sommaire les noms, entre autres, de Gaston Miron, Paul-Marie Lapointe, Gilles Hénault, Serge Garant et Marcel Dubé.

${ }^{12}$ Ibid., p. 11.

${ }^{13}$ Journal d'un inquisiteur, op. cit., p. 37 (quand elle n'est suivie d'aucune indication, l'édition du Journal utilisée dans les notes est celle de 1960).

${ }^{1+}$ Ibid., p. 29.

${ }^{15}$ Dans le Journal d'un inquisiteur, Leclerc utilise de façon interchangeable les expressions «Canadiens français » (pp. 13, 15), «Québec français » (pp. 24, 224) et «société québecoise » [sic] (p. 261). Il précise de cette façon ce qu'il entend par ces expressions et ce qui constitue l'objet de son investigation : «Le territoire de la culture française en Amérique, ce n'est pas la Colombie-Britannique, la Nouvelle-Écosse ou le Connecticutt [sic], c'est le Québec ; point final » (p. 262).

${ }^{16}$ Ibid., pp. 15, 17, 308. 
${ }^{17}$ Ibid., p. 275.

${ }^{18}$ Ibid., p. 16.

19) Ibid., p. 18.

20) Ibid., pp. 13, 22.

${ }^{21}$ Ibid., pp. 22, 59.

22 Ibid., pp. 54, 53.

${ }^{23}$ Ibid., pp. 15, 20.

${ }^{24}$ Ibid., p. 23.

25 Ibid., p. 255.

${ }^{26}$ Ibid., pp. 239, 235, 243.

${ }^{27}$ Ibid., p. 232.

${ }^{28}$ Ibid., pp. 233, 236.

29 Journal d'un inquisiteur (édition de 1974), op. cit., pp. 15-17.

${ }^{30}$ Journal d'un inqusiteur, op. cit., pp. 43, 242.

${ }^{31}$ Ibid., p. 57.

32 Ibid., p. 290 note 8.

${ }^{33}$ Ibid., p. 26.

${ }^{34}$ Ibid., p. 63.

${ }^{35}$ Ibid., p. 39.

${ }^{36}$ Ibid., pp. 86-87, 105, 26.

${ }^{37}$ On se rappellera que pour Platon, tout régime (oligarchique, démocratique, etc.) repose en définitive sur un certain «type de personnalité » qui est à la fois son produit et le nourrit ou lui donne son sens (ainsi de l'« homme démocratique » en démocratie : La république, 557b, traduction de Pierre Pachet, Paris, Gallimard, 1993, p. 428).

${ }^{38}$ Journal d'un inqusiteur, op. cit., p. 206.

39) Ibid., pp. $217,46$.

4) Ibid., pp. 304, 45.

${ }^{41}$ Ibid., pp. 46. 
t2 Gilles Leclerc, Limirisible Occident (dialogue de proscrits), Montréal, Éditions de l'Aube, 1958, p. 8.

${ }^{+3}$ Journal d'un inquisiteur, op. cit., p. 49.

${ }^{+4}$ Ibid., pp. 140 (note 1), 202.

${ }^{45}$ Ibid., p. 305.

to Ibid., pp. 19, 37, 68-69.

${ }^{47}$ Ibid., pp. 55-56.

48 "Prométhée ou Schweitzer », op. cit., p. 14.

49) Journal d'un inquisiteur, op. cit., p. 222.

5" "Prométhée ou Schweitzer », pp. 13-14.

${ }^{51}$ Journal d'un inquisiteur, op. cit., p. 226.

52 «Prométhée ou Schweitzer », p. 13 ; Journal d'un inquisiteur, op. cit., pp. 83, 124, 180.

${ }^{53}$ Journal d'un inquisiteur, op. cit., pp. 133, 195.

${ }^{5+}$ Ibid., p. 33.

55 «Prométhée ou Schweitzer», op. cit., p. 11.

${ }^{56}$ Ibid.

57 Journal d'un inquisiteur, op. cit., p. 137.

${ }^{58}$ "Prométhée ou Schweitzer», op. cit., p. 14.

5) Ibid., p. 10.

(il) Ibid., p. 7.

${ }^{61}$ Journal d'un inquisiteur, op. cit., pp. 303, 304.

62 Ibid., pp. 166, 196, 267.

${ }^{6.3}$ Ibid., pp. 222-223.

${ }^{6+}$ La question de la « nature » est abordée dans la prochaine section.

${ }^{65}$ « Prométhée ou Schweitzer », op. cit., p. 16.

(6) Journal d'un inquisiteur, op. cit., pp. 37-38. Une étude des sources qui ont inspiré Leclerc révélerait que sans qu'il ne les cite directement, il reprend 
souvent plusieurs thématiques développées par Dostoïevski, Nietzsche, Péguy, Mounier, Ortega y Gasset ou Spengler.

67 Ibid., p. 41.

${ }^{68}$ Ibid., pp. 90, 12.

69) Ibid., p. 222.

${ }^{70}$ Ibid., p. 198 ; « Prométhée ou Schweitzer », op. cit., p. 14.

${ }^{71}$ Journal d'un inquisiteur, op. cit., p. 25.

${ }^{72}$ Ibid., pp. 222, 229.

${ }^{73}$ Ibid., pp. 107, 175.

${ }^{74}$ Ibid., pp. 205, 120, 168, 259.

${ }^{75} \mathrm{Ibid}$., p. 135. On le voit, la critique féroce du régime théologico-politique ne conduit nullement Leclerc à rompre avec l'héritage classique et chrétien : même si le Journal d'un inquisiteur en particulier « donne l'impression de [s]'acquitter d'un devoir d'ingratitude envers la religion et la patrie », il reste que son auteur reconnait leur devoir « la meilleure partie de [s]a substance d'homme » (Ibid., p. 11).

${ }^{76}$ Ibid., pp. 33, 175.

${ }^{77}$ Ibid., pp. 237-238.

${ }^{78}$ Ibid., pp. 195-196.

${ }^{79}$ Ibid., pp. 137, 83.

${ }^{80}$ Pour le dire un peu paradoxalement : l'humanité est libre d'être libre, au sens de s'orienter vers l'Esprit, ou non.

${ }^{81}$ Ibid., p.126 ; Gilles Leclerc, " Préface », dans Paul Mercure, Les cris, Montréal, Éditions de l'Aube, 1957, p. 3.

82 Journal d'un inquisiteur, op. cit., p. 126.

${ }^{8.3}$ Ibid., pp. 106, 125.

${ }^{84}$ Ibid., p. 107.

${ }^{85}$ Ibid., p. 64.

${ }^{86}$ Ibid., pp. 84, 90.

${ }^{87}$ Ibid., p. 104. 
88 Ibid., p. 132.

${ }^{89}$ Ibid., p. 24.

"1) Ibid., p. 73.

${ }^{91}$ Ibid., p. 78.

${ }^{92}$ Ibid., pp. 73-74.

${ }^{93}$ Ibid., p. 29.

${ }^{94}$ Ibid., p. 81 ; voir aussi p. 195.

95 Ibid., p. 191.

"Ibid., pp. 179-180.

${ }^{97}$ Ibid., p. 60.

${ }^{98}$ Ibid., p. 46.

99) Ibid., p. 114.

${ }^{1011}$ Ibid., p. 189.

${ }^{1111}$ Ibid., p. 76.

1112 Ibid, pp. 247, 304 ("poïétique " = poièsis ?).

${ }^{103}$ Ibid., p. 117.

${ }^{104}$ Ibid., pp. 34, 73, 74.

${ }^{105}$ Ibid., p. 115.

1166 Ibid., p. 231.

1117 Ibid., p. 208.

${ }^{108}$ Ibid., p. 258.

1119) Ibid., p. 257.

1111 Ibid., pp. 164-165.

${ }^{111}$ Ibid., pp. 222, 258.

112 Ibid., p. 172.

${ }^{113}$ Gilles Leclerc. La chair abolie. Montréal, Éditions de l'Aube, 1957. 63 p.

${ }^{11+}$ Journal d'un inquisiteur, op. cit., p. 257.

115 Ibid., p. 270. 
${ }^{116}$ Ibid., pp. 75, 236, 13.

${ }^{117}$ Ibid., pp. 94, 155.

${ }^{118}$ Ibid., pp. 74, 180 (note 3).

119) Ibid., pp. 28, 35.

${ }^{120}$ Ibid., p. 19.

${ }^{121}$ Ibid., pp. 49, 193, 203.

${ }^{122}$ Ibid., p. 255.

${ }^{123}$ Ibid., p. 132.

${ }^{124}$ Ibid., p. 142.

${ }^{125}$ Ibid., p. 97.

${ }^{126}$ Ibid., p. 64.

${ }^{127}$ Ibid., p. 55.

${ }^{128}$ Ibid., p. 142.

${ }^{129}$ Ibid., p. 49 (les italiques sont dans le texte).

130) Ibid., p. 142.

${ }^{131}$ Linvisible Occident, op. cit.

${ }^{132}$ Sans parler du communisme, dont on trouve une critique dans L'invisible Occident, pour qui les sacrifices du présent ne sont que le prix à payer pour le bonheur de demain.

${ }^{133}$ Journal d'un inquisiteur, op. cit., p. 274.

${ }_{134}$ «Préface », dans Paul Mercure, Les cris, op. cit. p. 4.

135 «Prométhée ou Schweitzer», op. cit., p. 7.

${ }^{136}$ Journal d'un inquisiteur, op. cit., p. 49.

${ }^{137}$ Ibid., p. 305.

${ }^{138}$ Ibid., p. 101 (les italiques sont dans le texte).

139) «Préface », dans Paul Mercure, Les cris, op. cit. p. 5.

${ }^{140)}$ Ibid., p. 6.

${ }^{141}$ Journal d'un inquisiteur, op. cit., pp. 149, 151, 157. 
${ }^{1+2}$ "Préface », dans Paul Mercure, Les cris, op. cit. pp. 7-8.

${ }^{1+3}$ Journal d'un inquisiteur, op. cit., p. 247.

${ }^{14+}$ Ibid., p. 35.

${ }^{145}$ Ibid., p. 256 (note 5).

${ }^{1+6}$ Ibid., p. 159.

${ }^{147}$ Ibid., p. 298.

${ }^{1+8}$ Ibid., p. 288 (les italiques sont dans le texte).

14) Ibid., pp. 111-112.

${ }^{150}$ Ibid., p. 302. 